This item was submitted to Loughborough's Research Repository by the author.

Items in Figshare are protected by copyright, with all rights reserved, unless otherwise indicated.

\title{
Psychomotor performance decrements following a successful physical activity intervention for insomnia
}

PLEASE CITE THE PUBLISHED VERSION

https://doi.org/10.1080/15402002.2019.1578774

\section{PUBLISHER}

(C) Taylor \& Francis (Routledge)

\section{VERSION}

AM (Accepted Manuscript)

\section{PUBLISHER STATEMENT}

This is an Accepted Manuscript of an article published by Taylor \& Francis in Behavioral Sleep Medicine on 17 Feb 2019, available online: https://doi.org/10.1080/15402002.2019.1578774.

\section{LICENCE}

CC BY-NC-ND 4.0

\section{REPOSITORY RECORD}

Hartescu, luliana, Kevin Morgan, and Clare Stevinson. 2019. "Psychomotor Performance Decrements Following a Successful Physical Activity Intervention for Insomnia”. Loughborough University. https://hdl.handle.net/2134/36878. 


\section{ABSTRACT}

Evidence supports the view that reductions in cognitive hyperarousal contribute substantially to improved sleep outcomes following cognitive and behavioral interventions for insomnia disorder. Assuming an inverted-u relationship between arousal and performance, a theoretical possibility, supported by limited empirical data, is that the same mediating processes could negatively impact aspects of psychomotor performance, reducing speed on tests of reaction time.

Sedentary participants (mean age $=59.8 ; \mathrm{SD}=9.46)$ meeting research diagnostic criteria for insomnia were randomized to either an exercise intervention of $\geq 150$ min of moderate-intensity activity per week $(n=20)$, or a wait-list control group $(n=21)$. Of these, $n=17$ intervention and $n=18$ control participants completed 6-month follow-up assessments.

Digit span, and simple and complex vigilance task performance was assessed using a computerized protocol at baseline and 6-month follow-up. Dependent variables included digit span, simple reaction time (SRT), complex reaction time (CRT), false positive responses, number of lapses, and SRT/CRT ratio (indicative of the magnitude of difference between simple and complex RT performance). The primary clinical outcome was Insomnia Severity Index (ISI) score.

In comparisons of baseline to follow-up change, ISI scores showed clinically significant improvement in the intervention group at 6-month follow-up $(\mathrm{F}(8,26)=5.16 ; \mathrm{P}=0.03)$. Baseline vigilance performance was equivalent in both groups. At 6-month follow-up, however, the intervention group showed significantly slower simple reaction time $F(4,30)=10.25, \mathrm{p}<0.01$, and a significantly decreased SRT/CRT ratio $(\mathrm{F}(4,30)=13.22, \mathrm{p}<0.01)$.

Among people meeting diagnostic criteria for insomnia, beneficial sleep outcomes following successful behavioral interventions may, under some circumstances, come at the cost of slower psychomotor performance. 
INTRODUCTION

Compromised daytime functioning is frequently reported by insomnia patients (Shekleton., Rogers, \& Rajaratnam, 2014), and remains a prominent diagnostic criterion for insomnia disorder (American Psychiatric Association, 2013).

Nevertheless, inconsistent findings from controlled evaluations indicate that insomnia-performance relationships can be complex and subtle. In a seminal review of laboratory findings, for example, Riedel \& Lichstein (2000) concluded that, despite subjective complaints, people with insomnia “...do not suffer from significant daytime deficits". While some discrepancies between subjective and objective findings may be attributable to bias in patient reports (Orff, Drummond, Nowakowski, \& Perlis, 2007) or test insensitivity (Fortier-Brochu, Beaulieu-Bonneau, Ivers, \& Morin, 2012), evidence is emerging of a tangible pattern of measurable insomnia-related performance deficits. For example, on a range of standard neurocognitive tests insomnia has been associated with impairments of sustained and shifting attention (Altena, Van Der, Ysbrand, Strijers, \& Van Someren, 2008), deficits in episodic memory, and compromised decision making (see Fortier-Brochu et al, 2012). Consistent with these deficits, epidemiological studies have shown that insomnia increases the risk of in-home and road-traffic accidents (Leger et al, 2014; Garbarino et al, 2017), and sickness absenteeism (Daley, Morin, LeBlanc, Grégoire, \& Savard, 2009; Reynolds et al, 2017).

Inconsistencies in the evidence base might also reflect interactions between daytime performance and the insomnia phenotype. In controlled comparisons using 'simple' and 'complex' vigilance tasks, Altena et al. (2008) reported that reaction time performance for an insomnia group was significantly superior (i.e. faster) on the simple task, but significantly slower on the complex task (relative to non-insomnia 
controls). Similarly, in a secondary analysis of data from the UK Biobank project (where performance data were collected in study assessment centers), Kyle et al (2017) found that those reporting the highest frequency of insomnia symptoms also showed significantly better performance on tests of basic reaction time, and visual and prospective memory tests, after adjustments for demographic, medical and other sleep-related (e.g. sleep duration) variables. One possible explanation for these findings is that people with insomnia may recruit additional neurocognitive resources in order to match the demands of simpler tasks (Orff et al, 2007), but find it harder to keep pace as task complexity increases (Altena et al, 2008). This 'complexity cost' for people with insomnia is further suggested in test results involving functions such as working memory and decision-making processes (Edinger, Carney, \& Wohlgemuth, 2008; Fortier-Brochu et al, 2012). A 'complexity cost' hypothesis is also consistent with the arousal model of insomnia (Harvey, 2002; Espie, Broomfield, MacMahon, Macphee, \& Taylor, 2006; Riemann et al, 2010), whereby insomnia is conceptualized as a 24-hour hyperarousal disorder. Following an inverted-U model, hyperarousal could serve as both a facilitator of better performance on simple tasks, while contributing to impaired performance on more complex tasks.

Methodologically, these 'complexity cost' and 'arousal' hypotheses provide a framework for both testing and interpreting insomnia-related daytime performance: experimental tasks should differ in complexity and demand, while complexity costs can be captured in the ratio of less-complex to more-complex task performance (Altena et al, 2008). These hypothesized mechanisms have direct implications for treatment outcomes. If psychological treatments for insomnia are mediated by reductions in arousal, then phenotypically superior performance on simpler tasks may actually be impaired following successful interventions. To date, however, 
relatively little research has focused on post-treatment performance outcomes in insomnia treatment studies.

Building on epidemiological evidence significantly associating higher physical activity levels with better sleep quality (Youngstedt \& Kline, 2006; Buman \& King, 2010), and lower physical activity levels with elevated insomnia risk (Janson et al, 2001; Morgan, 2003) we designed and executed a randomized controlled trial of an exercise intervention for sedentary people meeting research diagnostic criteria for insomnia disorder (Hartescu et al, 2015). In the present analyses we compare the pre- and post-treatment cognitive performance of treated and control participants. In line with the arguments presented here, it was hypothesized that a successful exercise intervention for insomnia would: 1) significantly increase (i.e. impair) simple reaction time; and 2) significantly reduce the simple reaction time (in milliseconds) to complex reaction time (in milliseconds) ratio. A third hypothesis, that a successful exercise intervention for insomnia would reduce complex reaction time, was also tested. Finally, to assess whether any performance change resulted from gross cognitive differences not specific to attentional processes, performance on a Digit Span Task. was also compared. For this latter comparison, it was hypothesized that there would be no intergroup difference.

\section{MATERIAL AND METHODS}

\section{Design and intervention}

The host study was designed as a two-arm (activity intervention versus wait-list control) parallel randomized controlled trial. Assessments were conducted at baseline, and at 6 months post-baseline follow-up. The activity intervention commenced immediately after the baseline assessments, and ended at the post- 
baseline assessment. Participants in the intervention group were instructed to engage in brisk walking of at least moderate intensity, for a minimum duration of 150 minutes per week. Participants were further advised to distribute their walking over at least 5 days per week, choosing a setting (recreational walking, walking to work,

etc.) and a time of day which suited them best. In the control group, participants were asked to continue their lifestyle as usual, and maintain their existing levels of physical activity (see Hartescu et al, 2015, for a full description of the trial procedure). The study was approved by Loughborough University Ethics Approvals (Human Participants) Sub-Committee.

\section{Participants}

Participants $(\mathrm{N}=41,30$ females; mean age $=59.80$ years $)$ were randomized to the exercise intervention $(n=20)$ or the wait-list control $(n=21)$ groups, stratified by age and gender. Inclusion criteria were: men or women aged 40 years or older meeting Research Diagnostic Criteria for insomnia (Edinger et al, 2004); ambulant and independent in activities of daily living; moderate to vigorous intensity physical activity participation less than 30 minutes per day, on 5 or more days per week in the previous 6 months; and stable on any non-excluded medication taken over the previous 3 months. Exclusion criteria were: Epworth Sleepiness Scale (Johns, 1991) scores >10; reporting all 4 'essential diagnostic criteria for Restless Legs Syndrome' (Allen, Picchietti, Hening, Trenkwalder, Walters, and Montplaisir J (2003); diagnosis of any other sleeping disorder; history of cognitive or neurological disorders, or of any major psychiatric disorder; significant depressive symptoms as indicated by Beck Depression Inventory II score >31 (Kendall, Hollon, Beck, Hammen \& Ingram, 1987; Beck, Steer, and Carbin,1988); the presence of chronic disease for which moderate-intensity physical activity would be contraindicated; taking anti- 
hypertensive or hypolipidemic medication; engaged in shift work or other selfimposed irregular sleep schedules; a body mass index $(\mathrm{BMI})>35$; history of smoking over the previous 6 months; or caffeine consumption greater than $300 \mathrm{mg}$ per day. For women, being pre- or peri-menopausal and/or receiving estrogen replacement therapy also served as exclusion criteria. Of those randomized, $n=17$ intervention and $n=18$ control participants completed the 6-month follow-up assessments (see Figure 1).

\section{Sleep and Activity Outcomes}

For continuous 14-day periods at baseline and 6-month follow-up, sleep and physical activity were separately assessed using the Actiwatch 2 actigraph (Philips Respironics Inc, US) and the GTX3+ accelerometer (ActiGraph Corp, US) respectively. Actiwatch 2 data provided key sleep outcomes, while GTX3+ accelerometry supplied an instrumental measure of adherence. In addition to estimating sleep variables (total sleep, sleep latency and sleep efficiency) the Actiwatch 2 also incorporates a light sensor, allowing average daily light exposure (lux/min) to be calculated. Using published protocols (Troiano et al, 2008) GTX3+ data were divided above and below the threshold for 'moderate to vigorous physical activity' (MVPA) and expressed as the variable MVPA minutes per week.

Participant profilesIn addition to socio-demographic status, sleep and health status were profiled using: the Insomnia Severity Index (ISI; Morin et al, 1993; 2011); the Beck Depression Inventory (BDI-II; Beck et al, 1988); State-Trait Anxiety Inventory (STAl; Spielberger, Gorsuch, Lushene, Vagg, \& Jacobs, 1983); the Epworth Sleepiness Scale (ESS; Johns, 1991); and the Fatigue Severity Scale (FSS; Krupp et al, 1989). Health related quality of life was assessed using the self-completed 
EQ5D-5L (EuroQol Group, 1990), comprising 5 dimensions: mobility; self-care; usual activities; pain/discomfort; and anxiety/depression. Dimension scores were then used to calculate a Health Index Value, with higher values indicating better health. In addition, the EQ5D-5L includes a single visual Analogue scale (EQ VAS), where participants rate their health 'today' on a visual scale from 0 to 100 . BMI was calculated from self-reported height and weight.

\section{Performance tasks}

All performance tasks were administered using E-prime 1.1, with service pack 3 (Psychology Software Tools, Pittsburgh, PA, USA). During the tasks, test stimuli (Courier New bold font size 45) appeared in the middle of a $30.5 \times 23 \mathrm{~cm}$ LCD screen (screen resolution $640 \times 480$ ) against a light grey background. All tasks were administered within the two weeks of actigraphy sleep monitoring at baseline and 6month follow up period; in the participant's home, in a quiet room, in the afternoon (between 1pm and 7pm, keeping the same time for the baseline session and 6month follow-up sessions), and in the same order to all participants: digit span task, simple reaction task, and complex reaction.

\section{Digit span task}

During this task, a sequence of numbers would appear on the screen, one number at a time. When the sequence completed, the screen would return to blank. The participant was then asked to enter the number sequence using the keyboard, in the order it appeared on the screen. Digits were randomly sampled without replacement up to list lengths of 9 digits in a row (with single digit duplications when participants' spans surpassed 9). Successive digits could not occur in regular ascending or descending sequences with equal consecutive step sizes (e.g., 123, 876, 357, 864, 
or 369). The sequence span length was increased following correct answers and repeated following an incorrect response, up to maximum of 12 trials. The dependent variable for this task was the length of the longest correct span over the 12 trials.

\section{Simple and complex vigilance tasks}

Simple and complex vigilance performance was assessed using the computerized tasks fully described by Altena et al (2008). For the simple vigilance task, participants were presented with 110 sequential stimuli (a star symbol) appearing on the screen in the same location, but at random intervals of 1-10 seconds. Participants were instructed to respond to the stimuli on the screen by pressing the return key as quickly as possible. The task had a duration of approximately 13 minutes. For the complex vigilance task, either the target letter ' $p$ ', or the distractor letter 'd' would appear on the screen at random time intervals of 0.5 - 5 seconds. There was a brief training session of 10 stimuli (five targets) preceding the task. Participants were instructed to respond as accurately and as quickly as possible to the targets while ignoring the distracters. Participants were instructed to respond to the correct (target) stimuli on the screen by pressing the return key as quickly as possible. For both tasks a single 'trial' commenced with stimulus presentation and ended with the participant's response, or the expiry of a pre-defined lapse period. The following dependent variables were calculated: i) simple reaction time (SRT) and complex reaction time (CRT) for the simple/complex vigilance tasks respectively and quantified in milliseconds (ms); ii) number of lapses, defined as a response slower than $500 \mathrm{~ms}$ on the 'simple' vigilance task, or a response slower than $637 \mathrm{~ms}$ on the 'complex' vigilance task (this latter threshold was estimated empirically as $500 \mathrm{~ms}$ + the mean difference between the complex and simple vigilance task duration (which was $137 \mathrm{~ms}$ ); iii) false-positive responses to distractor 
stimuli on the complex vigilance task; and iv) the ratio of simple to complex reaction time (SRTms/CRTms). For the purposes of calculating mean SRT and CRT, responses scored as lapses were ignored. In all analyses, results from the first three target stimuli were discarded in order to eliminate start-up problems (resulting in 107 actual target stimuli for each test).

\section{Analyses}

Data were analyzed using SPSS version 20.0 (IBM, Armonk, NY, USA). All data were checked for outliers, distribution variance, and missing values. Statistical assumptions were assessed using standard normality tests. Statistical significance was defined as $p<.05$ using two tailed tests.

For the clinical outcome assessments, bias analyses were conducted for missing data from participants who were randomized, but dropped out before the first followup period. Where these analyses indicated that missing data biased the outcomes of the trial, multiple imputation or last observation carried forward methods were employed. For participants who completed performance tests at baseline and followup, there were no missing values.

Performance in the control and intervention groups was compared in analysis of covariance (ANCOVA) models where change (baseline minus follow-up) provided the dependent variable. Covariates were: baseline performance on the cognitive test; light exposure (min/day of light exposure over 1000 lux); Beck Depression Inventory score; and time of testing with three levels (1-3pm; 3-5pm; and 5-7pm). Assumptions of independence of the covariates and intervention effects, and homogeneity of regression slopes, were checked and met through a series of interaction univariate models, controlling for the main effects. 
Sample size was based on the post-treatment ISI scores, and assumed an effect size of 1.25 with beta at $20 \%$, alpha at $5 \%$, and an allocation ratio of $1: 1$ (see Hartescu et al, 2015). Effect sizes and confidence intervals were computed using the Effect Size Generator Professional Edition software (v. 4.1, ClinTools Software, Melbourne, Australia), by inputting adjusted means and standard deviations from SPSS for the respective variables.

\section{RESULTS}

\section{Baseline characteristics and clinical outcomes}

Recruitment and follow-up participation are shown in Figure 1. Participant characteristics at baseline are shown in Tables 1 and 2. None of the variables violated the equality of variance assumption, or normality of distributions assumption (for the t-tests). At 6 months post-baseline the intervention group showed significantly higher mean levels of MVPA compared to the control group (213 $\mathrm{min} /$ week $v 82 \mathrm{~min} /$ week). Relative to controls the intervention group also showed a significant improvement in ISI scores at the 6 month post-baseline assessment (mean ISI change: intervention group = -4.06 (SD: 4.99); control group -1.42 (SD: $3.42) ; F(1,28)=5.16, p=0.03)$, together with significant reductions in trait anxiety and depression scores, $F(6,28)=4.41, p=0.05$, and $F(6,28)=5.61, p=0.02$, respectively.

\section{Performance outcomes}

The group mean maximum digit span at baseline was 6; this remained unchanged at 6-month follow-up. The lowest value of the maximum digit span was 4 , both at baseline and post-intervention; therefore none of the participants met the criteria 
(maximum digit span length of 3 ) to be eliminated from the analyses. There was no significant effect of intervention/control group membership on performance on the digit span task, $F(4,30)=.05, p=0.82$.

Across all participants and conditions (baseline and 6-month follow-up), the complex vigilance task required approximately $137 \pm 12.2 \mathrm{~ms}$ more than the simple vigilance task to perform. There was a significant effect of treatment condition on the change scores for simple reaction time, $F(4,30)=10.25, p<0.01$. Examination of the group means revealed that the intervention group had significantly slower reaction time on the simple vigilance task post-intervention (at 6-month follow-up), compared to their baseline performance, $t(15)=3.67, p<0.01$. There was no significant effect of treatment condition on the performance on the complex reaction time, $F(4,30)=.01$, $p=0.94$ (Table 4). Individual simple reaction time values for the intervention and control groups at baseline and 6-month follow-up are shown in Figure 2.

In the ANCOVA models comparing baseline and 6-month follow-up performance calculated as the ratio between the simple reaction time and complex reaction time (SRTms/CRTms), there was a significant effect of treatment condition, $F(4,30)=13.22, p<0.01$ (Table 4; Figure 3). Follow-up comparisons for each group revealed a significant decrease in performance ratio time in the intervention group, $t(16)=-2.74, p=0.01$; whilst participants in the control group displayed an increase in their ratio performance time, $t(15)=-2.5, p=0.04$. There were no significant differences in the SRTms/CRTms performance ratio at baseline, $F(1,38)=2.25$, $p=0.14$ (Table 4). There was no significant effect of time of day of testing on either of the performance outcomes tested. 
In final exploratory analyses, possible linear relationships between SRT change scores and baseline to follow-up change in ISI scores and actigraphically measured total sleep time, were evaluated in separate partial correlations controlling for group membership. Neither analysis showed a significant correlation (ISI change and SRT change: $r=-05 ; p=.78 ;$ TST change and SRT change: $r=-.17 ; p=36$ )

\section{DISCUSSION}

Clinical outcomes from the present trial showed that among otherwise inactive individuals meeting research diagnostic criteria for insomnia, a minimum level of 150 min of moderate-intensity physical activity per week, validated by objective measures of MVPA, was associated with significantly reduced insomnia symptom severity, and significantly elevated mood. These improvements in sleep and mood were independent of average daily light exposure (Hartescu et al, 2015).

\section{Performance outcomes}

The performance analyses reported here show a pattern of results consistent both with the present hypotheses and an explanation in terms of modified arousal in the intervention group. This view is supported by several factors.

First, performance on the digit span task showed no post-intervention differences, with performance change specific to reaction time variables. Second, SRT was significantly slower for the intervention group at follow-up. And third, change in the SRTms/CRTms ratio, indicative of a reduced magnitude of difference between simple and complex RT, was also greatest (and significant) in the intervention group at follow-up. It seems reasonable to conclude, therefore, that among people meeting 
diagnostic criteria for insomnia, beneficial sleep outcomes following a successful exercise intervention may come at the cost of slower psychomotor performance.

These findings fit with a hyperarousal model of insomnia (Bonnet and Arand, 1997) which would parsimoniously link the intervention and the sleep and performance outcomes. In the present study, improved sleep outcomes are quite likely the result of arousal levels modified by the exercise intervention. The research literature for other conditions in which arousal mechanisms play a central role, such as generalized anxiety disorders and post-traumatic stress disorder, have also shown that physical activity programmes can reduce symptoms commonly associated with hyperarousal (Stathopoulou, Powers, Berry, Smits, \& Otto, 2006). Following the inverted-U model, optimal levels of psychomotor performance require optimal levels of arousal, with hyperarousal often associated with faster reaction times. The present results, therefore, agree with a conclusion that the physical activity intervention contributed to the restoration of effective attention regulation mechanisms through reduced arousal, which in turn impacted sleep and performance outcomes.

The present results for simple RT accord with those reported by Altena et al (2008) following 6-week programs of combined insomnia therapies (CBT, bright light exposure, body temperature manipulation, and physical activity) administered to a sample of people with insomnia. However, while Altena et al (2008) also found a post-treatment improvement for complex RT, the present study showed no differences in complex RT at 6-month follow up. Complex RT tasks require the recruitment of additional cognitive load; hyperarousal effects in insomnia would predict a slower reaction time on these tasks. These differences in outcome may be attributable, at least in part, to methodology, with the a substantially longer follow-up period in the present study. It is also possible that the complex reaction time task 
used may have marginal sensitivity in heterogeneous samples of people with insomnia. The task required differentiating between the letters ' $p$ ' and ' $d$ '. Recent reviews of cognitive deficits in people with insomnia suggest that more complex tests may be needed to reliably capture change in samples of people with insomnia; significant impairment in people with insomnia, for example, is more apparent with tests of working and episodic memory, and problem-solving (Fortier-Brochu et al, 2012). These impairments better correspond to complaints of daytime performance from people with insomnia: poor concentration, memory difficulties, and inability to maintain attention on tasks (Ohayon \& Lemoine, 2004).

Limitations of the present analyses include the sample sizes in the two groups, as the study was powered for a different main outcome (i.e. insomnia severity). However, the robust analyses employed, combined with the effect sizes reported, allow for conclusions to be drawn with confidence. That cognitive testing in the present study was not conducted under laboratory conditions might also be considered a limitation. The tests were administered in the participants' own home, in a quiet room, maintaining the same time of the day between 1-7pm, and using the same equipment. In addition, measures were taken to ensure protocol adherence, and minimize 'noise'. To an unknown extent, the advantages of familiar surroundings, and the convenience of being visited by the experimenter could actually have contributed to the validity of measured performance. A further limitation of the present study is the absence of intervening performance tests between baseline and 6-month follow-up. A compromise between 'ideal' data requirements, and participant tolerance, the frequency of all participant contacts was carefully planned over the duration of the trial. As a result, we cannot speculate on whether psychomotor performance oscillated and stabilized, or steadily changed 
over the follow-up period. Finally, since the present randomized controlled trial was designed to assess treatment outcomes, the inclusion of a 'non insomnia' control was not considered appropriate. As a result, the influence of phenotypic arousal levels on baseline psychomotor performance is assumed (Fortier-Brochu and Morin, 2014). In relation to this point, we also acknowledge that, in excluding those with insomnia disorder and high levels of daytime sleepiness (i.e. ESS scores $>10$ ) we may have biased the sample in favor of those with higher levels of baseline arousal. Future trials could usefully include more frequent psychomotor testing sessions, and an additional control group comprising non-insomnia participants. Furthermore, results from the exploratory correlations, though not definitive, also suggest that change in simple reaction time values was not a linear function of either ISI or TST change, indicating that a more detailed evaluation of sleep quality-RT relationships is warranted.

\section{AUTHOR CONTRIBUTIONS}

IH contributed to study design, data collection, data analyses, interpretation of results and preparation of the manuscript. KM and CDS contributed to study design, data analyses, interpretation of results and preparation of the manuscript.

\section{CONFLICTS OF INTEREST}

$\mathrm{IH}, \mathrm{KM}$ and CDS have no conflict of interest to disclose.

\section{ACKNOWLEDGEMENTS}

We thank Professor Eus JW Van Someren for giving us permission to use the psychomotor vigilance tasks. 


\section{References}

Allen R P, Picchietti D, Hening W A, Trenkwalder C, Walters A S, Montplaisir J. Restless legs syndrome: diagnostic criteria, special considerations, and epidemiology . A report from the restless legs syndrome diagnosis and epidemiology workshop at the National Institutes of Health. Sleep Med 2003; 4(2):101-119.

Altena, E., Van Der, W., Ysbrand, D., Strijers, R. L. M., \& Van Someren, E. J. W. (2008). Sleep loss affects vigilance: effects of chronic insomnia and sleep therapy. Journal of sleep research, 17(3), 335-343.

American Psychiatric Association. (2013). Diagnostic and statistical manual of mental disorders (5th ed.). Washington, DC: Author.

Beck, A. T., Steer, R. A., \& Carbin, M. G. (1988). Psychometric properties of the Beck Depression Inventory: Twenty-five years of evaluation. Clinical Psychology Review, 8(1), 77-100.

Bonnet, M. H., \& Arand, D. L. (1997). Hyperarousal and insomnia. Sleep medicine reviews, 1(2), 97-108.

Daley, M., Morin, C. M., LeBlanc, M., Grégoire, J.-P., \& Savard, J. (2009). The economic burden of insomnia: direct and indirect costs for individuals with insomnia syndrome, insomnia symptoms, and good sleepers. Sleep, 32(1), 55-64.

Edinger, J. D., Bonnet, M. H., Bootzin, R. R., Doghramji, K., Dorsey, C. M., Espie, C. A., . . Stepanski, E. J. (2004). Derivation of research diagnostic criteria for insomnia: report of an American Academy of Sleep Medicine Work Group. Sleep, 27(8), 1567-1596.

Edinger, J. D., Carney, C. E., \& Wohlgemuth, W. K. (2008). Pretherapy cognitive dispositions and treatment outcome in cognitive behavior therapy for insomnia. Behavior Therapy, 39(4), 406-416.

Espie, C. A., Broomfield, N. M., MacMahon, K., Macphee, L. M., \& Taylor, L. M. (2006). The attention-intention-effort pathway in the development of psychophysiologic insomnia: a theoretical review. Sleep Medicine Reviews, 10(4), 215-245.

EuroQol Group. (1990). EuroQol-a new facility for the measurement of health-related quality of life. Health Policy, 16, 199-208.

Fortier-Brochu, É., Beaulieu-Bonneau, S., Ivers, H., \& Morin, C. M. (2012). Insomnia and daytime cognitive performance: a meta-analysis. Sleep Medicine Reviews, 16(1), 8394.

Fortier-Brochu, É., \& Morin, C. M. (2014). Cognitive impairment in individuals with insomnia: clinical significance and correlates. Sleep, 37(11), 1787-1798.

Garbarino, S., Magnavita, N., Guglielmi, O., Maestri, M., Dini, G., Bersi, F. M., .. . Durando, P. (2017). Insomnia is associated with road accidents. Further evidence from a study on truck drivers. PLoS ONE, 12(10), e0187256. doi:10.1371/journal.pone.0187256 
Hartescu, I., Morgan, K., \& Stevinson, C. D. (2015). Increased physical activity improves sleep and mood outcomes in inactive people with insomnia: a randomized controlled trial. Journal of sleep research.

Harvey, A. G. (2002). A cognitive model of insomnia. Behaviour research and therapy, 40(8), 869-893.

Johns, M. W. (1991). A new method for measuring daytime sleepiness: The Epworth Sleepiness Scale. Sleep, 14(6), 540-545.

Krupp, L. B., LaRocca, N. G., Muir-Nash, J., \& Steinberg, A. D. (1989). The fatigue severity scale: Application to patients with multiple sclerosis and systemic lupus erythematosus. Archives of Neurology, 46(10), 1121.

Kyle, S. D., Sexton, C. E., Feige, B., Luik, A. I., Lane, J., Saxena, R., . . Little, M. A. (2017). Sleep and cognitive performance: cross-sectional associations in the UK Biobank. Sleep medicine, 38, 85-91.

Leger, D., Bayon, V., Ohayon, M. M., Philip, P., Ement, P., Metlaine, A., . . Faraut, B. (2014). Insomnia and accidents: cross-sectional study (EQUINOX) on sleep-related home, work and car accidents in 5293 subjects with insomnia from 10 countries. Journal of sleep research, 23(2), 143-152.

Morin, C. M., Barlow, D. H., \& Dement, W. C. (1993). Insomnia: Psychological assessment and management (treatment manuals for practitioners). New York: The Guilford Press.

Morin, C. M., Belleville, G., Bélanger, L., \& Ivers, H. (2011). The insomnia severity index: Psychometric indicators to detect insomnia cases and evaluate treatment response. Sleep, 34(5), 601-608.

Ohayon, M. M., \& Lemoine, P. (2004). Daytime consequences of insomnia complaints in the French general population. Encephale, 30(3), 222-227.

Orff, H. J., Drummond, S. P. A., Nowakowski, S., \& Perlis, M. L. (2007). Discrepancy between subjective symptomatology and objective neuropsychological performance in insomnia. Sleep, 30(9), 1205-1211.

Reynolds, A. C., Appleton, S. L., Gill, T. K., Taylor, A. W., McEvoy, R. D., Ferguson, S. A., \& Adams, R. J. (2017). Sickness absenteeism is associated with sleep problems independent of sleep disorders: results of the 2016 Sleep Health Foundation national survey. Sleep Health, 3(5), 357-361. doi:10.1016/j.sleh.2017.06.003

Riedel, B. W., \& Lichstein, K. L. (2000). Insomnia and daytime functioning. Sleep Medicine Reviews, 4(3), 277-298.

Riemann, D., Spiegelhalder, K., Feige, B., Voderholzer, U., Berger, M., Perlis, M., \& Nissen, C. (2010). The hyperarousal model of insomnia: a review of the concept and its evidence. Sleep Medicine Reviews, 14(1), 19-31.

Shekleton, J. A., Rogers, N. L., \& Rajaratnam, S. M. (2010). Searching for the daytime impairments of primary insomnia. Sleep Medicine Reviews, 14(1), 47-60. 
Spielberger, C. D., Gorsuch, R. L., Lushene, R., Vagg, P. R., \& Jacobs, G. A. (1983). Manual for the state-trait anxiety inventory. Palo Alto, CA: Consulting Psychologists Press.

Stathopoulou, G., Powers, M. B., Berry, A. C., Smits, J. A. J., \& Otto, M. W. (2006). Exercise interventions for mental health: a quantitative and qualitative review. Clinical Psychology: Science and Practice, 13(2), 179-193.

Troiano, R. P., Berrigan, D., Dodd, K. W., Mâsse, L. C., Tilert, T., \& McDowell, M. (2008). Physical activity in the United States measured by accelerometer. Medicine and Science in Sports and Exercise, 40(1), 181-188. 


\section{Table 1}

Participant socio-demographic characteristics at baseline

\begin{tabular}{llll}
\hline Variable & $\begin{array}{l}\text { Sample } \\
\mathrm{N}=41\end{array}$ & $\begin{array}{l}\text { Control group } \\
\mathrm{N}=21\end{array}$ & $\begin{array}{l}\text { Intervention } \\
\text { group } \mathrm{N}=20\end{array}$ \\
& & Mean (SD) or frequency \\
\hline Age & $59.80(9.46)$ & $60.10(8.51)$ & $59.50(10.59)$ \\
Gender (female/male) & $30 / 11$ & $15 / 6$ & $15 / 5$ \\
Education (high/low) & $17 / 24$ & $9 / 12$ & $8 / 12$ \\
Marital status (married/not) & $36 / 15$ & $12 / 9$ & $14 / 6$ \\
Income groups (median) & 7.00 & 6.50 & 9.00 \\
Body mass index & $26.29(4.18)$ & $26.58(4.77)$ & $25.99(3.55)$ \\
MVPA* min/week & $57.50(30.07)$ & $48.95(27.84)$ & $66.50(30.37)$ \\
\hline
\end{tabular}

* MVPA=Moderate to vigorous physical activity 


\section{Table 2}

Participant self-reported sleep and health characteristics at baseline

\begin{tabular}{llll}
\hline Variable & $\begin{array}{l}\text { Sample } \\
\mathrm{N}=41\end{array}$ & $\begin{array}{l}\text { Control group } \\
\mathrm{N}=21\end{array}$ & $\begin{array}{l}\text { Intervention } \\
\text { group N }=20\end{array}$ \\
& $16.11(4.93)$ & $16.37(5.58)$ & $15.82(4.23)$ \\
& $11.87(6.51)$ & $12.95(6.84)$ & $10.75(6.13)$ \\
ISI $^{\mathrm{a}}$ & $39.95(8.73)$ & $40.14(9.68)$ & $39.74(8.09)$ \\
BDI $^{\mathrm{b}}$ & $34.75(7.98)$ & $35.71(8.52)$ & $33.68(7.41)$ \\
STAI $^{c}$-Trait & $77.63(16.53)$ & $75.24(20.82)$ & $80.15(10.28)$ \\
STAI $^{\mathrm{c}-S t a t e}$ & $3.96(1.53)$ & $4.04(1.54)$ \\
EQ-5D (VAS) & $3.51)$ & $3.88)$ \\
FSS & & & \\
\hline
\end{tabular}

${ }^{\mathrm{a}} \mathrm{ISI}=$ Insomnia Severity Index; ${ }^{\mathrm{b}} \mathrm{BDI}=$ Beck Depression Inventory; ${ }^{\mathrm{c}} \mathrm{STAI}=$ State/Trait Anxiety Inventory; ${ }^{\mathrm{A}} \mathrm{FSS}=$ Fatigue Severity Scale 


\section{Table 3}

Simple and complex vigilance task performance pre- and post-intervention (baseline and 6-month follow-up values)

Reaction time values

\section{Mean \pm SD}

Performance measure

All sample Control Intervention $\mathrm{p}^{*}$

$$
\mathrm{N}=35 \quad \mathrm{~N}=18 \quad \mathrm{~N}=17
$$

SRT (ms) baseline

$365.97 \pm 42.73$

$369.89 \pm 70.97$

$362.04 \pm 45.12$

0.56

SRT (ms) post-intervention

$378.38 \pm 35.75$

$365.66 \pm 29.67$

$389.75 \pm 38.92$

CRT (ms) baseline

$487.92 \pm 41.23$

$482.42 \pm 44.46$

$493.43 \pm 38.07$

0.40

CRT (ms) post-intervention

$499.78 \pm 32.86$

$498.39 \pm 32.42$

$501.25 \pm 34.25$

SRT/CRT ratio at baseline

$1.34 \pm .14$

$1.31 \pm .11$

$1.37 \pm .16$

0.12

SRT/CRT ratio post-

$1.33 \pm .14$

$1.36 \pm .11$

$1.29 \pm .16$

intervention

SRT Lapses (n) at baseline

$7.27 \pm 4.04$

$7.05 \pm 4.35$

$7.50 \pm 3.80$

0.88

SRT Lapses (n) post-

$8.00(3.95)$

$7.88 \pm 3.80$

$8.11 \pm 4.22$

intervention

CRT lapses (n) at baseline

$10.42 \pm 9.07$

$9.10 \pm 7.25$

$11.75 \pm 10.62$

0.68

CRT lapses (n) post-

$9.11 \pm 4.26$

$8.55 \pm 3.69$

$9.70 \pm 4.83$

intervention

CRT false positives at

$2.15 \pm 1.29$

$2.30 \pm 1.39$

$2.00 \pm 1.07$

baseline

CRT false positives post-

$1.65 \pm 1.23$

$1.16 \pm .98$

$2.17 \pm 1.28$

intervention 
*Independent t-tests between groups at baseline; equality of variance tested and $\mathrm{n} / \mathrm{s}$ in all tests 


\section{Table 4}

Change in simple and complex vigilance task performance pre- and post-intervention (baseline value minus 6-month follow-up value).

\begin{tabular}{|c|c|c|c|c|c|}
\hline \multirow[b]{2}{*}{ Performance measure } & \multicolumn{2}{|c|}{ Mean \pm SD } & \multirow[b]{2}{*}{$\mathrm{p}$} & \multirow[b]{2}{*}{ Adjusted mean difference $\pm \mathrm{SE}(95 \% \mathrm{CI})$} & \multirow[b]{2}{*}{$\begin{array}{l}\text { Adjusted Cohen's d } \\
(95 \% \mathrm{CI})\end{array}$} \\
\hline & Intervention group & Control group & & & \\
\hline Simple RT (ms) & $-31.98 \pm 35.85$ & $4.65 \pm 30.32$ & 0.003 & $-30.19 \pm=9.42(-49.44--10.93)$ & $-1.1(-1.78--0.36)$ \\
\hline Complex RT (ms) & $-13.58 \pm 30.56$ & $-8.18 \pm 28.01$ & $0.94^{\prime}$ & $-4.58 \pm 9.18(-23.50-14.32)$ & $-.17(-0.82-0.48)$ \\
\hline Ratio SRTms/CRTms & $.09 \pm .12$ & $-.04 \pm .07$ & 0.001 & $-.11 \pm .03(-.18--.04)$ & $1.09(0.35-1.83)$ \\
\hline
\end{tabular}

Groups compared in ANCOVA models; covariates: Baseline value of performance measure; Beck Depression inventory score; light exposure (avg. min/day); time of testing. 


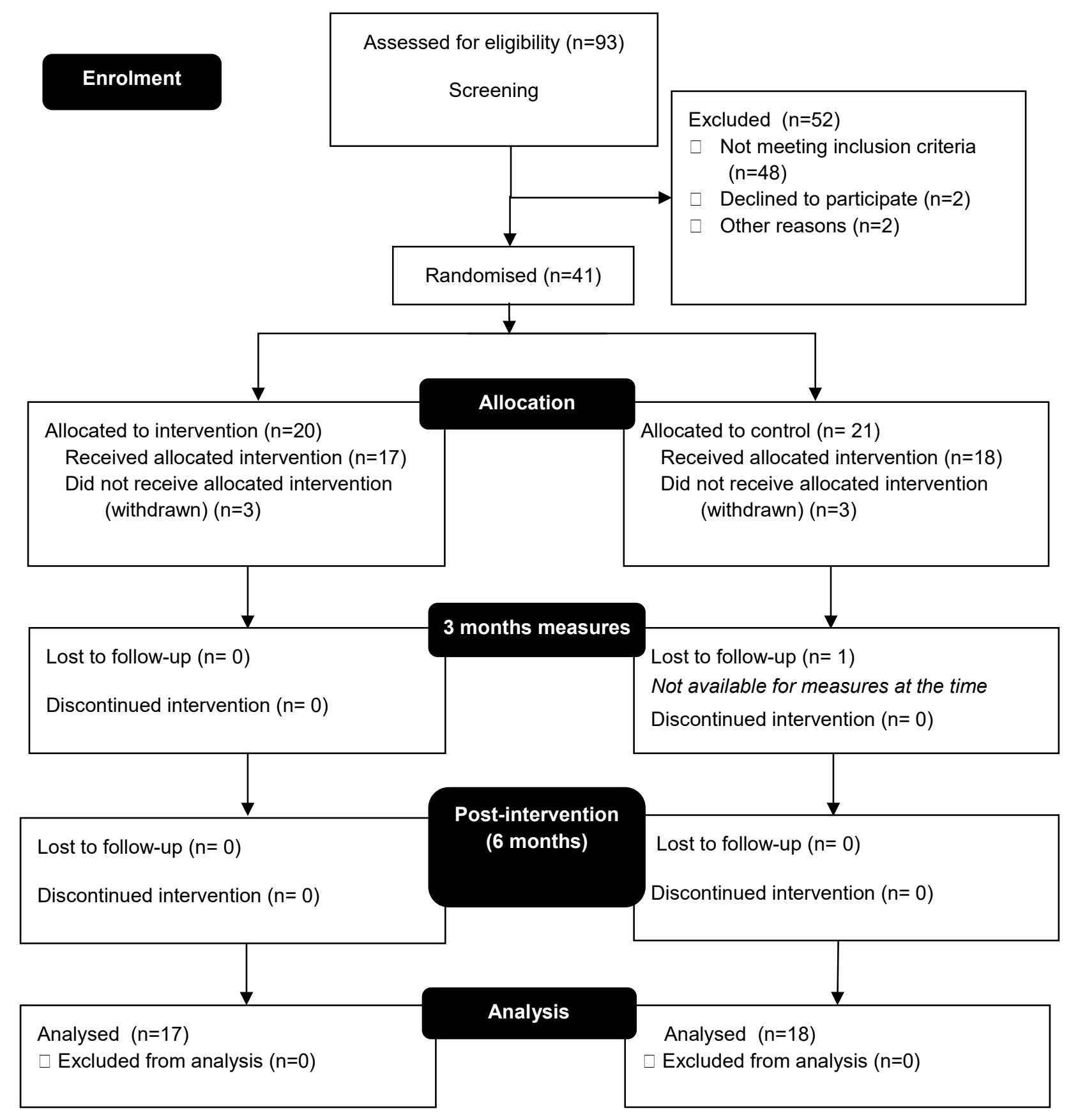

Figure 1: Participant recruitment flow diagram of the randomised controlled trial. 


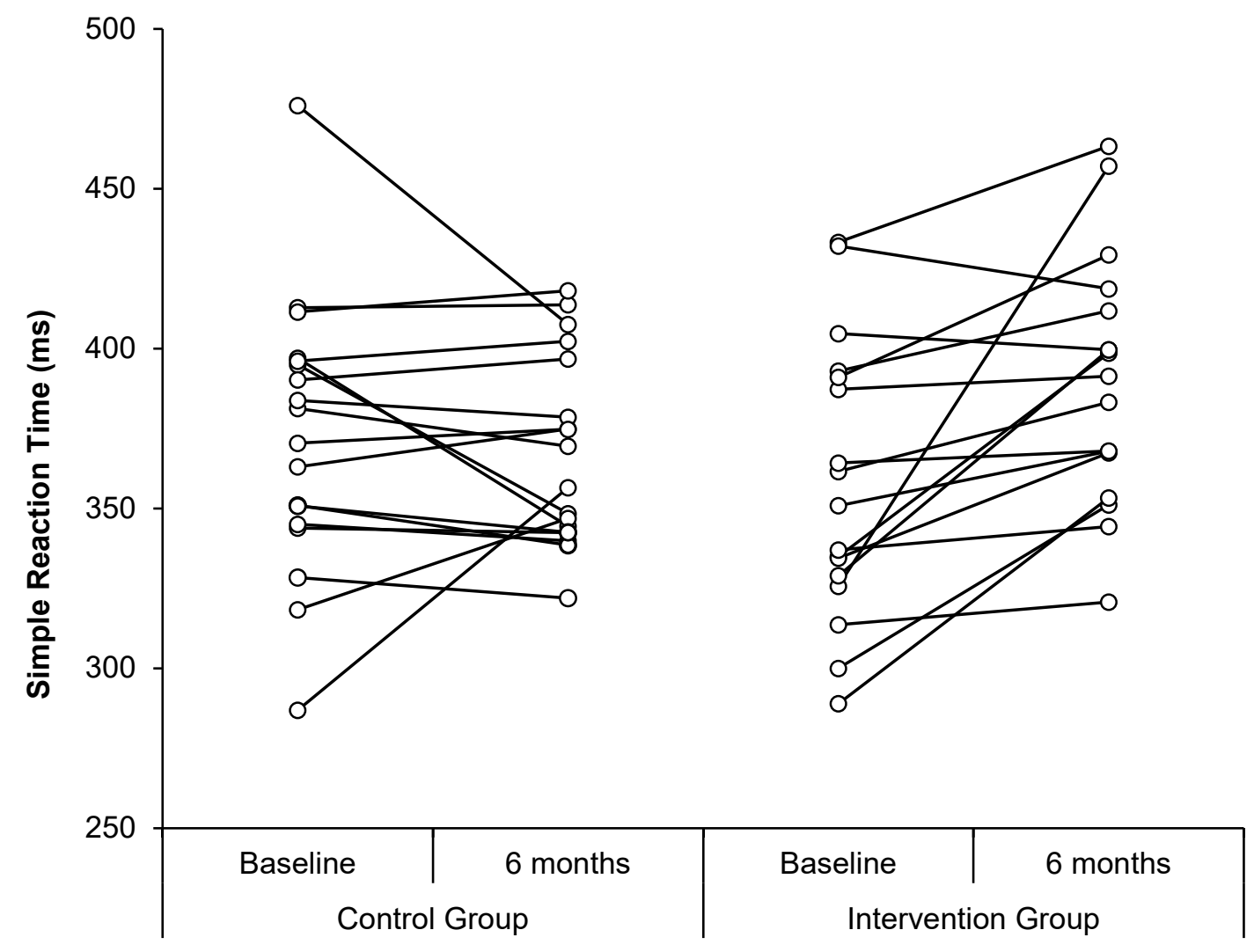

Figure 2. Individual simple reaction time values at baseline and 6-month follow-up 


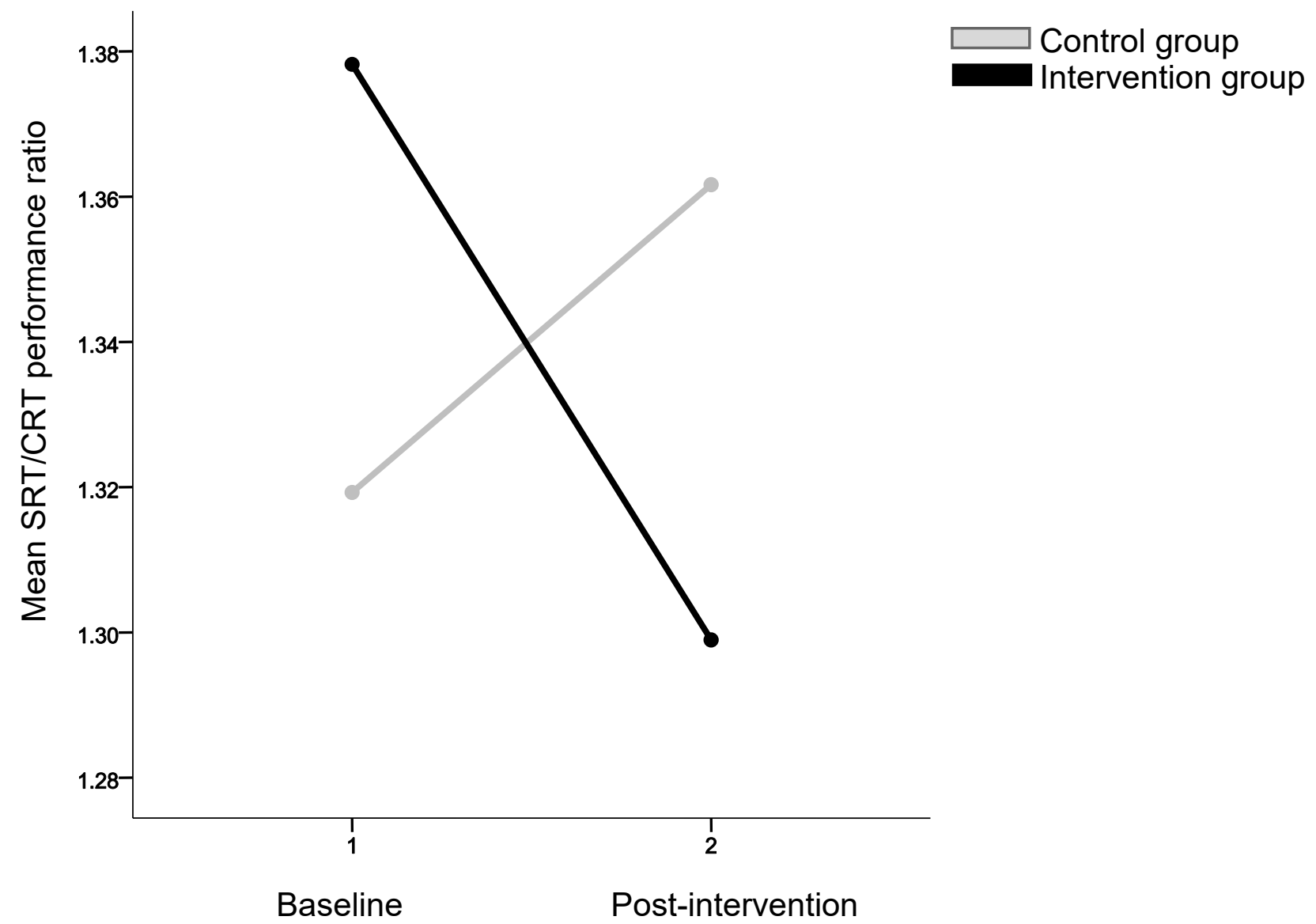

Figure 3. Mean SRTmsec/CRTmsec ratio change between baseline and 6-month follow-up in the two groups 